\title{
Efectos de la aplicación de tratamientos isotérmicos sobre el tamaño de grano y la dureza de aleaciones de aluminio AA5083-H116
}

\section{Effects of isothermal treatments application on grain size and hardness of AA5083-H116 aluminum alloy}

Jimy Unfried-Silgado ${ }^{1}$, Julieth López del Río ${ }^{2}$ Dinayra Machuca Vargas ${ }^{2}$, Ellery Pérez Caicedo ${ }^{1}$,Carlos Zambrano Pinedo ${ }^{1}$, Enrique Esteban Niebles Núñez ${ }^{1}$

\footnotetext{
${ }^{1}$ Programa de Ingeniería Mecánica. Grupo de Investigación en Materiales, Procesos y Tecnologías de Fabricación - IMTEF, Universidad Autónoma del Caribe. Calle 90 No.46-112, Bl. G, piso 2. ZIP 080020. Barranquilla, Colombia e-mail: j.unfried.s@gmail.com

${ }^{2}$ Programa de Ingeniería Industrial. Grupo de Investigación en Materiales, Procesos y Tecnologías de Fabricación - IMTEF, Universidad Autónoma del Caribe. Calle 90 No.46-112, Bl. G, piso 2. ZIP 080020. Barranquilla, Colombia e-mail: eniebles@uac.edu.co
}

\section{RESUMEN}

En este trabajo se estudia la relación entre las características morfológicas del grano y la dureza de la aleación de aluminio AA5083-H116 cuando se somete a la acción de tratamientos isotérmicos diversos no acumulativos. Para el análisis se usaron las técnicas de microscopia óptica y electrónica de barrido, microanálisis químico, medición de durezas, barridos de calorimetría diferencial, cálculos con termodinámica computacional y análisis de imágenes con el objetivo de estimar las temperaturas de inicio de la recristalización y de crecimiento de grano, así como las transformaciones de fase asociadas a estos ciclos térmicos. Se determinó que la temperatura de inicio de recristalización estática del material está entre $150^{\circ}$ y $180^{\circ} \mathrm{C}$, ocurriendo como un proceso heterogéneo, mientras que un posible crecimiento anormal del grano inicia aproximadamente a $\operatorname{los} 300^{\circ} \mathrm{C}$. Estos resultados son importantes para entender lo que ocurre en la microestructura durante los procesos de fabricación en los cuales se presenten ciclos térmicos similares, como en soldadura por fusión y tratamientos térmicos aplicados sobre este tipo de material y su relación con los cambios en propiedades locales.

Palabras-clave: Aleación de aluminio, recristalización, ciclos térmicos, tamaño de grano, DSC

\begin{abstract}
In this work is studied the relationship between morphological characteristics of grain and hardness of aluminum alloys AA5083-H116, which has been submitted to several isothermal treatments. For analysis development were used optical and scanning electron microscopy, chemical microanalysis, hardness measurements, differential scanning calorimetry (DSC) curves, computational thermodynamics calculations, and image digital analysis, aiming to determine the recrystallization and abnormal grain growth temperatures, as well as phase transformations associated to thermal cycles. It was determined that static recrystallization start temperature is between $150^{\circ}$ to $180^{\circ} \mathrm{C}$, which was observed as heterogeneous process at whole rolled structure of material, while a possible abnormal grain growth process is initiated at $300^{\circ} \mathrm{C}$, approximately. The results of this work are important because helping to understand the microstructure evolution due to processes of fabrication, which have similar thermal cycles, such as welding and heat treatments applied on this kind of material.
\end{abstract}

Keywords: Aluminum Alloys, recrystallization, thermal cycles, grain size, DSC

\section{INTRODUCCIÓN}


El aluminio es el material metálico no ferroso más consumido por la industria metalmecánica en todo el mundo [1]. El Aluminio y sus aleaciones son materiales metálicos con estructura cúbica centrada en las caras (FCC), con alta ductilidad, alta energía de falla de apilamiento y baja - media temperatura de fusión (alrededor de $\left.\operatorname{los} 660^{\circ} \mathrm{C}\right)$. Las aleaciones de aluminio son ampliamente utilizadas en la fabricación estructural, en substitución del acero en diversidad de industrias especializadas, tales como aeroespacial, naval y automovilística, gracias a su alta relación resistencia / peso y capacidad de conformación, en una amplia variedad de combinaciones microestructurales y tratamientos térmicos [2,3]. Las aleaciones de aluminio conformadas se dividen básicamente en dos grandes familias: tratables y no tratables térmicamente. Según el sistema de designación de aleaciones de aluminio, las aleaciones $2 \mathrm{xxx}, 6 \mathrm{xxx}$ y $7 \mathrm{xxx}$, pertenecen a la primera familia $\mathrm{y}$ usualmente se les denomina como aleaciones endurecidas por precipitación o por envejecimiento; mientras que las aleaciones de las series 1xxx, 3xxx y 5xxx pertenecen al segundo tipo, siendo conocidas como endurecidas por deformación plástica. Las aleaciones de la serie 4xxx varían según la composición química entre una y otra familia. La designación de la serie $8 \mathrm{xxx}$ se usa para aleaciones de aluminio especiales, mientras que la designación 9xxx no se utiliza comercialmente [4]. Los tratamientos termomecánicos más comunes aplicados a las aleaciones de aluminio son expresados mediante una letra mayúscula seguida de dos o tres dígitos. La designación de los tratamientos más usuales es: F, que identifica a la estructura como de fabricación sin tratamiento; $\mathrm{O}$, recocido; $\mathrm{H}$, endurecida por deformación; $\mathrm{W}$, tratamiento térmico de solubilización y $\mathrm{T}$, tratamiento térmico para producir estructuras estables [1].

Los aluminios de la serie 5xxx son esencialmente aleaciones de aluminio y magnesio (Al-Mg) endurecidas por dos mecanismos, solución sólida y deformación, las cuales son utilizadas generalmente en construcción naval (cascos y superestructuras) y de recipientes de almacenamiento. El Magnesio en las aleaciones de aluminio de la serie 5xxx cuyo contenido no supera 5\% en peso, entra en solución sólida permitiendo un aumento apreciable de la resistencia a la tracción (entre 150 y $250 \mathrm{MPa}$ ), conservando una aceptable ductilidad próxima al $25 \%$ [5]. El tratamiento $\mathrm{H}-116$, especial para aleaciones con contenidos máximos de $5 \%$ en peso de $\mathrm{Mg}$, confiere alta resistencia mecánica a través del endurecimiento por deformación general y buena resistencia a la corrosión [5]. Los procesos de laminación y extrusión, comúnmente usados para obtener láminas y perfiles, respectivamente, deforman la microestructura, alargando los granos, dejando en su interior suficiente energía interna que se convierte en el potencial (driving force) para diversos procesos metalúrgicos posteriores. La serie A5xxx con tratamiento H116 tiene como característica principal en su microestructura, el almacenamiento de energía interna debido a la formación, multiplicación y acumulación de dislocaciones; entonces, la deformación permanente atribuida a este mecanismo es altamente susceptible de modificarse por la acción del calor [2].

La energía de falla de apilamiento del aluminio puro está entre 160 a $250 \mathrm{~mJ} . \mathrm{m}^{-2}$, lo cual favorece el movimiento de dislocaciones por deslizamiento (slip) durante la deformación, facilitando los procesos de deslizamiento cruzado (Cross-slip), disminuyendo la distancia entre dislocaciones parciales y beneficiando la formación de una subestructura de grano (subgrano) o células de dislocaciones lo que, finalmente favorece la recristalización [6]. No obstante, los elementos de aleación pueden disminuir el valor de la energía de falla de apilamiento. Por lo anterior, cuando una aleación de aluminio policristalina de la serie AA5xxx deformada se somete a ciclos isotérmicos se favorecen los procesos de recristalización y crecimiento de grano (normal y anormal) mucho más que la recuperación, dependiendo de los picos de temperatura y sus tiempos de sostenimiento aplicados [6,7]. Así mismo, la extensión de los efectos de la recristalización estática, produciendo granos mayores, será proporcional al nivel de energía interna almacenada, la intensidad de los ciclos térmicos aplicados y su duración [7]. En el primer caso, la nucleación y crecimiento de nuevos granos refinados se presenta, ocurriendo cambios en las propiedades mecánicas en el material. En el segundo caso, el crecimiento normal favorecido por la orientación relativa de los contornos y el crecimiento anormal a partir de los granos existentes, producen una reducción de la dureza y resistencia a la tracción del material [7,8]. Los procesos antes mencionados son la fuente de alteración de propiedades del material cuando este se expone a fuentes de calor localizadas o generalizadas, tal como ocurre durante los ciclos térmicos inherentes a los procesos de soldadura por fusión en la región de la zona afectada por el calor o por otro tipo de fuentes de energía usados en procesos termomecánicos y tratamientos térmicos $[9,10]$.

El objetivo de este trabajo es evaluar los efectos sobre la dureza y el tamaño de grano al aplicar diversos ciclos isotérmicos sobre la estructura de una aleación de aluminio naval de la serie AA5083 con tratamiento H-116 en estado de entrega. El análisis del tamaño de grano se evaluó desde su modificación morfológica. La discusión y el análisis se centran en las relaciones entre los cambios morfológicos en la estructura, variación de la dureza y los fenómenos de recuperación, recristalización y crecimiento de grano estáticos como resultado de los ciclos isotérmicos aplicados. 


\section{MATERIALES Y MÉTODOS}

\subsection{Materiales y obtención de muestras}

Las muestras analizadas en este trabajo fueron obtenidas a partir de una lámina de 4,6 mm de espesor de la aleación AA5083-H116. El material fue sometido a análisis por espectrometría de emisión óptica para determinar su composición química. A partir del material laminado se extrajeron muestras de 10x10x4,6 $\mathrm{mm}^{3}$ utilizando una cortadora automática refrigerada (Metkon ${ }^{\circledR} /$ Metacut-M 250) cuidando que la temperatura de corte no sobrepasará los $40^{\circ} \mathrm{C}$. La localización de las muestras extraídas corresponde a las secciones transversal (T), longitudinal (L) y normal (N) al sentido de laminación de la placa, como se muestra en la Figura 1(b). Cada muestra se identificó marcándola con un código alfanumérico usando herramientas Gravuren ${ }^{\circledR}$ de $5 \mathrm{~mm}$.

\subsection{Simulación termodinámica computacional}

Usando la composición química obtenida experimentalmente y el programa de termodinámica computacional basado en el método Calphad, Thermo-Calc $\mathbb{R} 3.1$ fueron calculadas a través de simulaciones numéricas las transformaciones de fase en equilibrio de la aleación AA5083-H116, utilizando la base de datos TTAL-7 de Thermo-Tech ${ }^{\circledR}$. Se obtuvieron diagramas pseudobinarios en función del \%-peso de $\mathrm{Mg}$ y el de fracción de fases presentes en función de la temperatura para la aleación estudiada. Estos diagramas servirán como punto de partida para estudiar lo que ocurre durante los ciclos isotérmicos aplicados.

\subsection{Aplicación de tratamientos isotérmicos}

El material cortado y debidamente identificado fue sometido a una serie tratamientos isotérmicos no acumulativos como el mostrado en la Figura 1(a), donde cada muestra fue colocada dentro de un horno tipo mufla de marca Terrígeno ${ }^{\circledR}$ Modelo FPCA serie 593 e isotérmicamente tratada durante un tiempo de $600 \mathrm{~s}(10 \mathrm{mi}$ nutos) a la temperatura de sostenimiento $\left(\mathrm{T}_{\mathrm{ex}}\right)$, para luego enfriarla súbitamente (temple) en agua calma a $25^{\circ} \mathrm{C}$. Para cada temperatura $\left(\mathrm{T}_{\mathrm{ex}}\right)$, se trataron en un solo lote tres muestras correspondientes a las secciones de corte descritas en la obtención de muestras, como se muestra en la Figura 1(b). Las temperaturas de sostenimiento y la identificación de cada muestra se resumen en la Tabla 1.

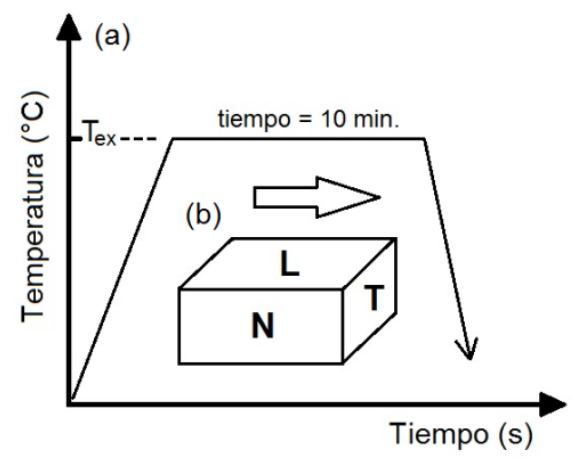

Figura 1: Esquemas de: (a) ciclos térmicos aplicados y (b) corte de las muestras. La flecha indica el sentido de laminación. L: Longitudinal, N: Normal y T: Transversal.

\subsection{Caracterización del material y análisis microestructural}

Luego de tratadas y debidamente marcadas, las muestras fueron montadas en resina epóxica Epoxi/Harder ${ }^{\circledR}$ usando una relación resina/endurecedor de 4:1, con un tiempo de curado de $24 \mathrm{~h}$ a temperatura ambiente. Se realizó preparación metalográfica estándar usando papeles de lija con granulometrías 100, 240, 320, 400, 600, 1000, 1200 y 1500. Luego se realizó pulido usando Alúmina con granulometría de 0,3 y 0,5 $\mu$ m en una Pulidora Metkon-Forcipol 2V® a velocidades de rotación entre 150 y $200 \mathrm{rpm}$, constantemente refrigeradas con agua. El pulido final fue realizado con alúmina en disolución acuosa con tamaño de partícula de $0,05 \mu \mathrm{m}$ usando $200 \mathrm{rpm}$ de rotación.

Tabla 1: Identificación de muestras y temperaturas experimentales de ciclos térmicos aplicados.

\begin{tabular}{c|c|c|c|c|c|c|c}
\hline Identificación de serie ensayada & $\mathbf{1}$ & $\mathbf{2}$ & $\mathbf{3}$ & $\mathbf{4}$ & $\mathbf{5}$ & $\mathbf{6}$ & $\mathbf{7}$ \\
\hline Temperatura sostenimiento $-\mathrm{T}_{\mathrm{ex}}\left({ }^{\circ} \mathrm{C}\right)$ & 50 & 100 & 150 & 180 & 200 & 300 & 500 \\
\hline
\end{tabular}


Para el ataque químico se usaron como reactivos ácido fluorhídrico HF al 10\% en volumen y Poulton's $\left(2 \mathrm{ml} \mathrm{HF}+3 \mathrm{ml} \mathrm{HCl}+20 \mathrm{ml} \mathrm{HNO}_{3}+175 \mathrm{ml}\right.$ de agua destilada), sumergiendo cada muestra durante 360 $\mathrm{s}$ mientras se mantuvo la solución a $60^{\circ} \mathrm{C}$. Posteriormente, cada muestra atacada fue lavada y enjuagada con abundante agua corriente durante $240 \mathrm{~s}$. Muestras del material sin tratamiento térmico fueron analizadas usando un microscopio electrónico de barrido con cañón de campo de emisión, SEM Quanta 650FEG y se identificaron las fases intermedias con microanálisis químico EDS-INCA, Oxford Instruments X-Max ${ }^{\circledR}$ usando energías entre $25 \mathrm{kV}$ y entre 2 a $5 \mathrm{kV}$, respectivamente.

La microestructura de cada muestra tratada fue explorada a través de un microscopio óptico Olympus ${ }^{\circledR}$ GX-51 obteniéndose imágenes en diferentes magnificaciones $(20 x, 50 x$ y 100x). Las imágenes fueron digitalizadas y tratadas con el programa analizador de imágenes Image-J®. Los métodos normalizados de medición del tamaño de grano presentan restricciones para materiales altamente deformados, parcial o totalmente recristalizados y el interés de este trabajo es establecer la relación del cambio del tamaño de grano con la temperatura de tratamiento isotérmico [11]. En este trabajo se ha propuesto medir el tamaño de grano en los diferentes estados, a partir del análisis de imágenes. Para ello se realizó la medida bidimensional del tamaño de grano estableciendo, con ayuda del analizador de imágenes y para cada grano, en un área de $500 \mathrm{x}$ $500 \mu \mathrm{m}^{2}$ la mayor longitud en el sentido de laminación (largo) y la mayor longitud en el sentido transversal al de la laminación (ancho) (ver figura 2). Se realizó un muestreo de estas dos dimensiones en un total de 30 medidas por cada muestra y condición. Con los datos anteriores se obtuvieron gráficos de frecuencia de tamaño de grano, aplicando este proceso a todas las condiciones mostradas en la Tabla 1. Se adoptó como criterio de clasificación de las etapas de la alteración del tamaño de grano que, cuando se observa la reducción de las dos dimensiones medidas experimentalmente, esta es coherente con la recristalización primaria y cuando las dos dimensiones comienzan a crecer luego de esta primera etapa, es coherente con el crecimiento del grano, tal como lo expresa la literatura. En esta última condición se observó con especial cuidado la forma del borde de grano [6-8].

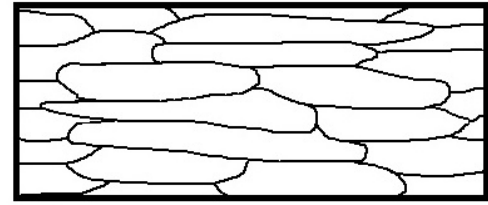

(a)

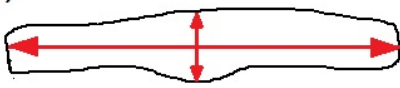

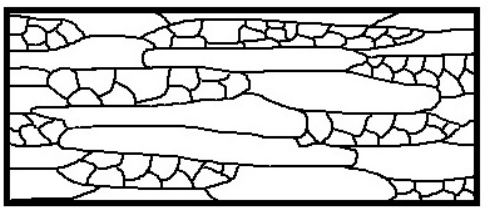

(b)

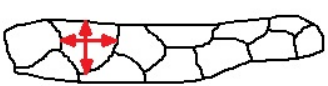

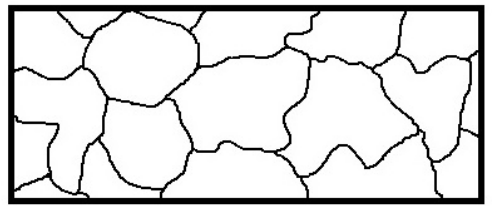

(c)

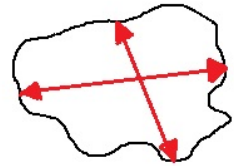

\section{Temperatura}

Figura 2: Esquema de la medida bidimensional del tamaño de grano en las diferentes etapas del tratamiento isotérmico. (a) En el estado de entrega. (b) A temperaturas bajas - intermedias. (c) A altas temperaturas.

A las muestras para cada condición tratada se les midió la dureza en la escala Vickers usando un durómetro Zwick ${ }^{\circledR}$ ZHU-187.5 y una carga de $3000 \mathrm{~g}$ durante $15 \mathrm{~s}$. Tres réplicas de cada medida se realizaron por cada orientación y condición. Las propiedades de tracción del material sin tratamiento térmico fueron analizadas usando una máquina Shimadzu ${ }^{\circledR}$ UH-100A usando una velocidad de $4 \mathrm{~mm} . \mathrm{min}^{-1}$ determinando la deformación durante el ensayo en una longitud libre de 50mm (2”).

\subsection{Barridos de calorimetría diferencial (DSC)}

Muestras independientes del material con composición química descrita en la Tabla 1 y en la condición de entrega fueron sometidas a análisis con la técnica de calorimetría de barrido diferencial (DSC), con el fin de obtener curvas de energía contra temperatura de la primera corrida para detectar picos de liberación (exotérmicos) o absorción (endotérmicos) de energía en el intervalo de temperaturas evaluado. El análisis de los picos fue asociado a procesos como recuperación, recristalización y transformaciones de fase. Se usó un equipo TA-Instruments ${ }^{\circledR}$ SDTQ600 DSC/DTA, bajo las siguientes condiciones: gas inerte $\mathrm{N}_{2}$ con un flujo de $100 \mathrm{ml} \cdot \mathrm{min}^{-1}$, rampas de calentamiento y enfriamiento de $10^{\circ} \mathrm{C} \cdot \mathrm{min}^{-1} \mathrm{e}$ intervalo de temperaturas experimentales entre $50^{\circ}$ y $600^{\circ} \mathrm{C}$. Las muestras evaluadas mediante esta técnica fueron laminillas de 9 a 15 mg provenientes de la lámina original cortadas con abundante refrigeración, cuidado extremo y lentitud para evitar el 
calentamiento excesivo y transformaciones no deseables.

\section{RESULTADOS}

\subsection{Caracterización del material en estado de entrega}

En la tabla 2 se muestra la composición química del material en estado de entrega obtenida experimentalmente. La composición confirma que el material trabajado pertenece a una aleación de aluminio AA5083 [1,2]. Los valores de propiedades mecánicas medidas experimentalmente fueron comparados con los observados en la literatura, siendo correspondientes con el tratamiento H116 para este tipo de aleación Al-Mg, como se observa en la tabla 3 [2].

Tabla 2: Composición química en porcentaje en peso de la aleación de aluminio AA5083-H116

\begin{tabular}{c|c|c|c|c|c|c|c|c|c}
\hline Elemento & Al & $\mathbf{M g}$ & $\mathbf{M n}$ & $\mathbf{F e}$ & $\mathbf{S i}$ & $\mathbf{C u}$ & $\mathbf{Z n}$ & $\mathbf{C r}$ & $\mathbf{N i}$ \\
\hline \%-peso & Balance & 4,017 & 0,418 & 0,238 & 0,022 & 0,176 & 0,013 & 0,061 & 0,015 \\
\hline \multicolumn{7}{c}{ D: Determinación realizada por Espectrometría de emisión óptica. }
\end{tabular}

Tabla 3: Valores de dureza, límite elástico y resistencia a la tracción determinados experimentalmente.

\begin{tabular}{c|c|c|c}
\hline Dureza Vickers & Límite elástico $\mathbf{S}_{\mathbf{y}} \mathbf{( M P a )}$ & Resistencia máxima $\left.\mathbf{S}_{\mathrm{ut}}(\mathbf{M P a})\right)$ & Alargamiento (\%) \\
\hline $95 \pm 2$ & $243 \pm 9,5$ & $337,5 \pm 16,5$ & 12,3 \\
\hline
\end{tabular}

La microestructura de la aleación estudiada en este trabajo fue examinada en su condición de entrega antes de la aplicación de tratamientos isotérmicos. En la figura 3 se observa la microestructura en las direcciones longitudinal, transversal y normal a la laminación, exhibiendo granos alargados y estrechos en la dirección del laminado del material como producto de la deformación aplicada al material.
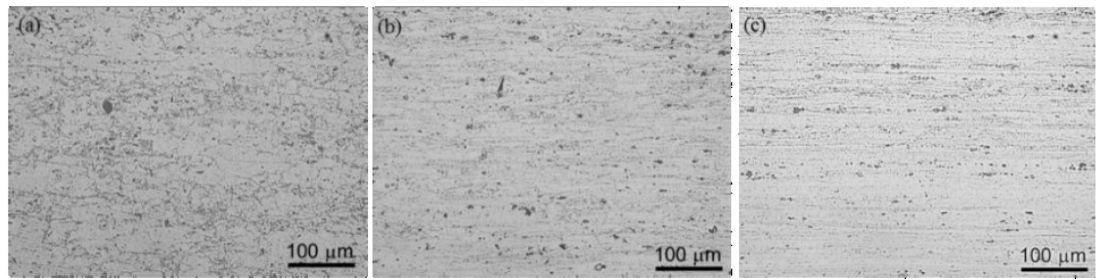

Figura 3: Microestructura del aluminio AA5083-H116 sin tratar. Sección (a) normal (N), (b) transversal (T) y (c) Longitudinal (L). Microscopia óptica. Ataque ácido fluorhídrico 10\%-vol. y Poulton's.

Además de la matriz rica en $\mathrm{Al}$ y $\mathrm{Mg}$, fueron observados, algunos precipitados dispersos en la matriz con técnicas de microscopia electrónica combinadas con microanálisis químico, encontrándose partículas redondeadas ricas en $\mathrm{Al}, \mathrm{Fe}$ y $\mathrm{Mn}$, dispersas en la estructura, mientras partículas cubiformes ricas en $\mathrm{Al}$ y $\mathrm{Mg}$ se encontraban a lo largo del contorno de grano, como se observa en la figura 4. Se determinó que las partículas ricas en $\mathrm{Al}$ y $\mathrm{Mg}$ son correspondientes con fases intermedias del tipo $(\beta) \mathrm{Al}_{3} \mathrm{Mg}_{2}$, mientras las otras son correspondientes con los tipos $\mathrm{Al}_{6}(\mathrm{Mn}, \mathrm{Fe})[1,2]$. 


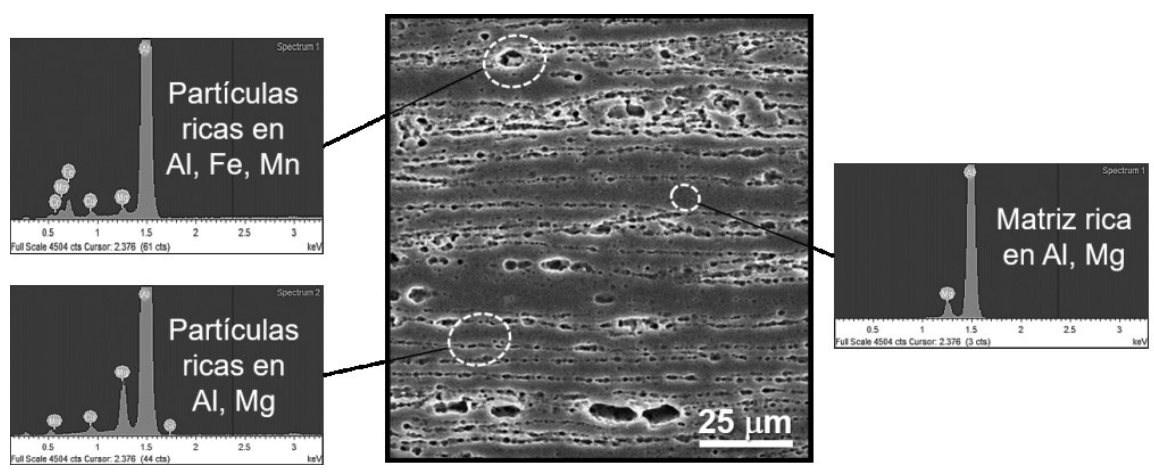

Figura 4: Identificación de fases intermedias y de la matriz en el material sin tratamiento. Imágenes de microscopia electrónica de barrido (SEM) y microanálisis químico (EDS). Ataque: Poulton's.

\subsection{Análisis de transformaciones de fase en equilibrio}

En la Figura 5 se muestran los diagramas de fracción de fases en equilibrio y el pseudobinario de los campos de equilibrio de fases en función de la temperatura calculados mediante el método Calphad, para la composición mostrada en la Tabla 1 . Se observa que el proceso de solidificación comienza a $639^{\circ} \mathrm{C}$ y finaliza a $589^{\circ} \mathrm{C}$, con un intervalo de solidificación de $50^{\circ} \mathrm{C}$. A temperatura ambiente son estables las fases: $\mathrm{FCC}(\gamma)$, correspondiente con la matriz de $\mathrm{Al}$ rico en $\mathrm{Mg}$ en solución sólida, con $87 \%$ de fracción másica; seguido aparece la fase intermedia $\mathrm{Al}_{3} \mathrm{Mg}_{2}(\beta)$ con aproximadamente $11 \%$ en fracción y dos fases intermedias $\mathrm{Al}_{6} \mathrm{Mn}$ y $\mathrm{Al}_{3} \mathrm{Fe}$, con $1,5 \%$ y $0,5 \%$, respectivamente. Como fases críticas dentro del intervalo de ciclos térmicos aplicados aparecen las fases $\mathrm{Al}_{3} \mathrm{Mg}_{2}$ y $\mathrm{Al}_{3} \mathrm{Fe}$, las cuales, por su temperatura de precipitación en equilibrio $\left(238^{\circ} \mathrm{C} \mathrm{y}\right.$ $119^{\circ} \mathrm{C}$, respectivamente), podrían ser susceptibles a sufrir disolución durante el calentamiento dependiendo del tiempo de permanencia y temperatura pico alcanzada durante los ciclos térmicos. Al comparar el diagrama binario Al-Mg con el pseudobinario de la aleación AA5083, se pone de manifiesto el efecto de las adiciones de $\mathrm{Mn}, \mathrm{Fe}$ y $\mathrm{Cu}$ sobre el campo de estabilidad de las fases intermedias $\mathrm{Al}_{6} \mathrm{Mn}$ y $\mathrm{Al}_{3} \mathrm{Fe}$.
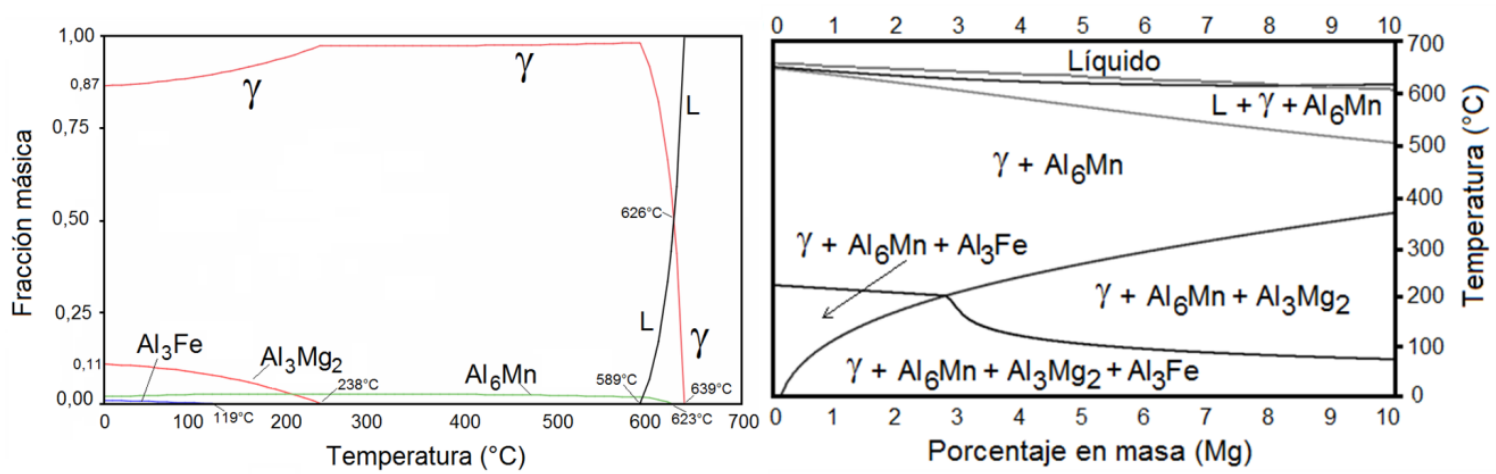

Figura 5: Diagrama de fracción másica de fases contra temperatura para la aleación AA5083-H116 estudiada.

\subsection{Evolución microestructural y análisis de durezas}

En la Figura 6 se observa una secuencia de imágenes que ilustra el cambio de la morfología del grano para algunas temperaturas de aplicación del tratamiento isotérmico aplicado y sus respectivas medidas de distribución de tamaños de grano en términos de la frecuencia porcentual, las cuales fueron obtenidas a partir del análisis de imágenes. Se detecta una importante reducción de las dimensiones del grano alrededor de las temperaturas entre $150^{\circ} \mathrm{C}$ y $200^{\circ} \mathrm{C}$, aunque de forma heterogénea.

En la Figura 7 se muestra el gráfico del comportamiento de las dimensiones ancho y largo con respecto a la temperatura de tratamiento. Se observa una reducción importante del tamaño de grano medido en la dirección longitudinal dentro del intervalo de temperaturas entre $100^{\circ} \mathrm{C} \mathrm{y} 180^{\circ} \mathrm{C}$, indicando que el proceso de recristalización primaria ha iniciado. Por la diferencia entre las dos dimensiones estabilizarse, se presume que ha ocurrido este proceso de manera heterogénea en el material. 
La evolución de las durezas en las diferentes direcciones del material, mostrada en la Figura 8, es coincidente con la evolución del tamaño de grano del material, donde la mayor dureza es observada en el intervalo entre $100^{\circ} \mathrm{C} \mathrm{y} 180^{\circ} \mathrm{C}$. Luego se observa un descenso en el valor de la dureza, siendo la reducción más drástica a partir de $300^{\circ} \mathrm{C}$.
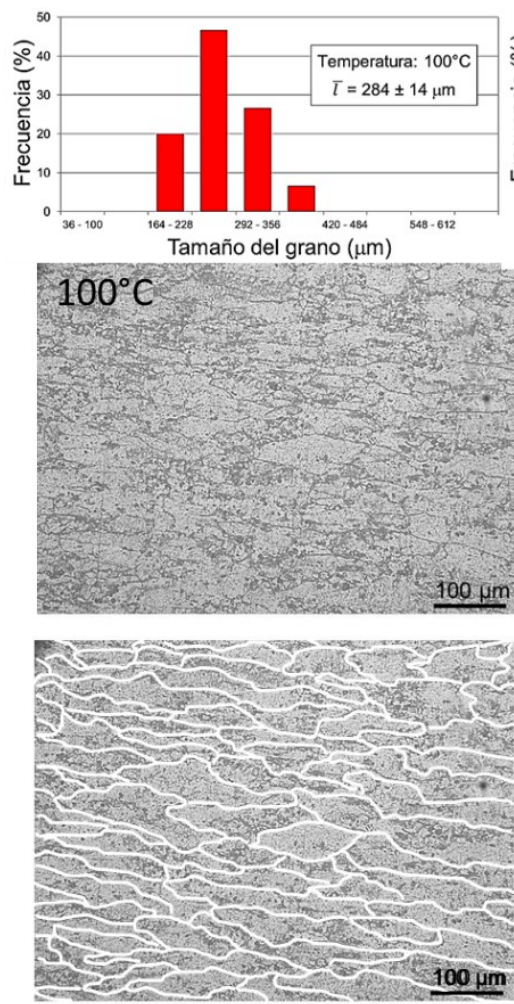

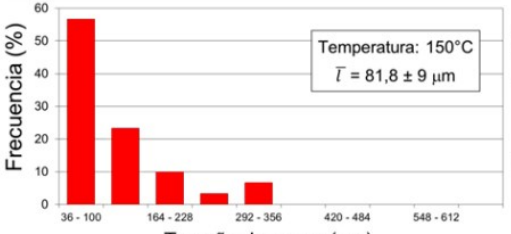

Tamaño de grano $(\mu \mathrm{m})$
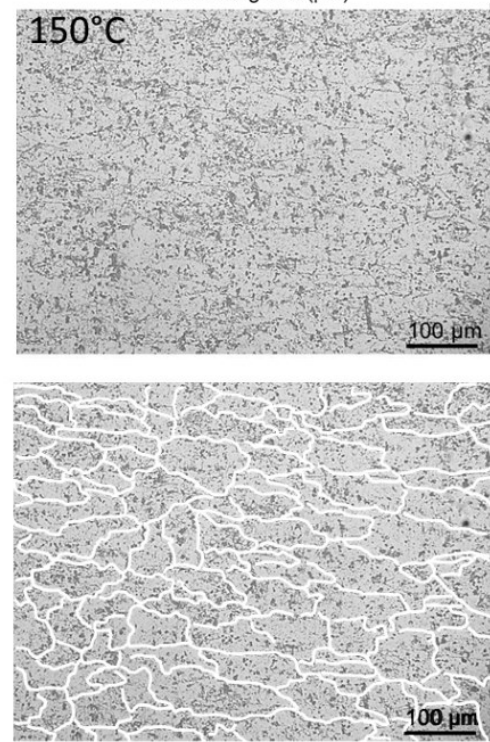
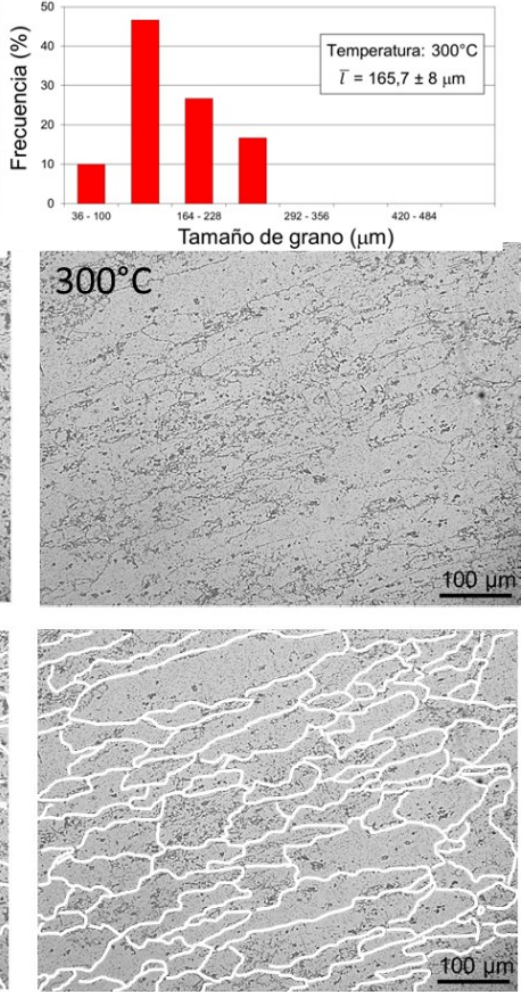

Figura 6: Evolución de la dimensión longitudinal (largo) del grano en función de la temperatura. Arriba: Gráficos de frecuencia porcentual del tamaño de grano. Medio: Imágenes de microestructura a diversas temperaturas. Abajo: Superposición del delineamiento de límite de grano y la microestructura por análisis de imágenes. Ataque: Poulton's.

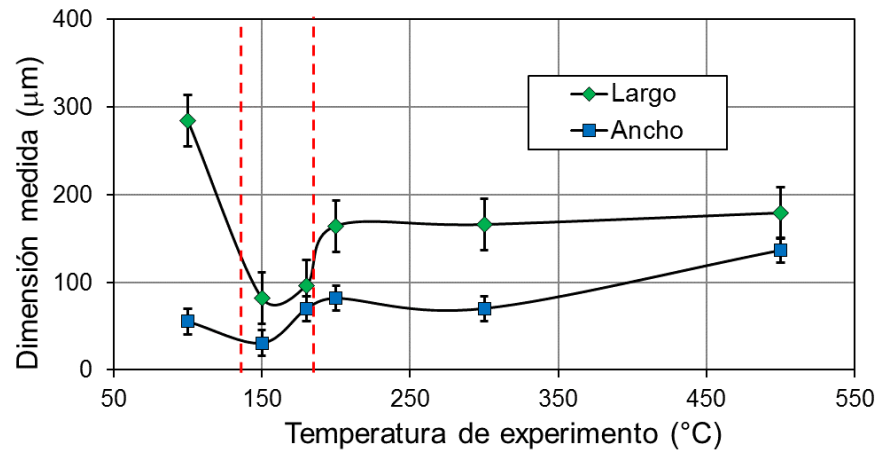

Figura 7: Evolución de las dimensiones ancho y largo del grano en función de la temperatura de experimento. 


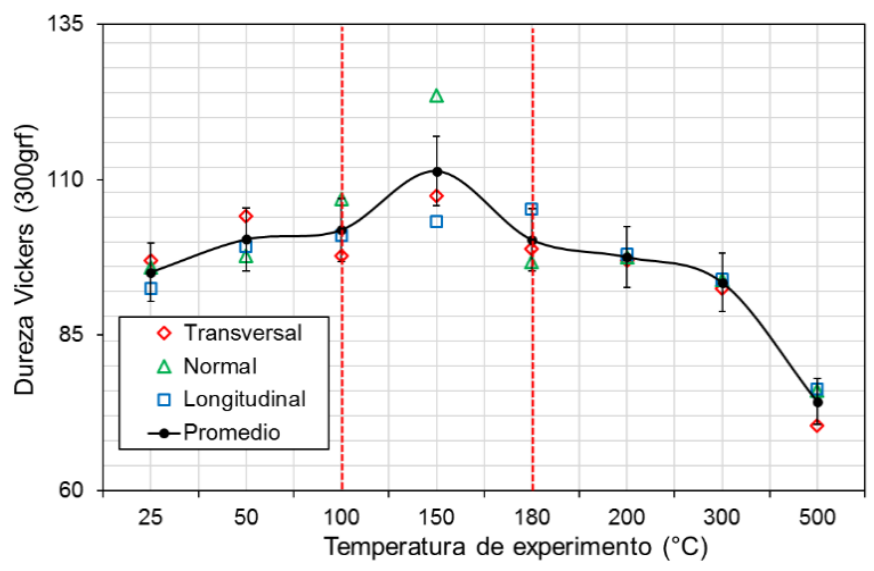

Figura 8: Comportamiento de la dureza en función de la temperatura de experimento por cada ciclo térmico.

Finalmente, el resultado de tres termogramas DSC independientes son mostrados en la figura 9, mostrando una fuerte reacción endotérmica entre $50^{\circ} \mathrm{C}$ y $180^{\circ} \mathrm{C}$.

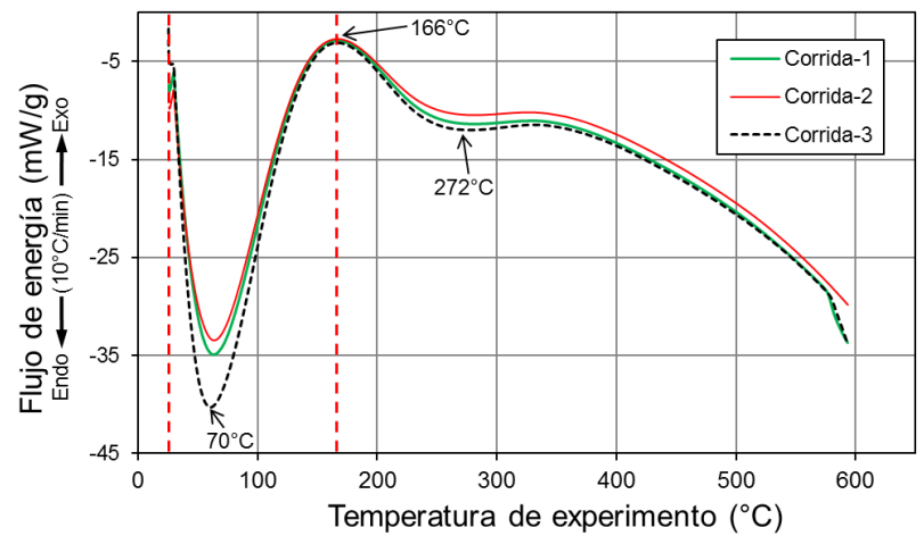

Figura 9: Barridos experimentales de calorimetría diferencial del material AA5083.

\section{DISCUSIÓN}

El análisis de la caracterización del material base, confirma que el material en estudio se trata de un aluminio AA5083-H116. El porcentaje de Mg se encuentra dentro del intervalo especificado, confiriendo alto endurecimiento por solución sólida y buena resistencia a la corrosión a este tipo de aleaciones incluso después del tratamiento termomecánico. La dureza media del material medida experimentalmente fue de $95 \pm 2 \mathrm{HV}$ en estado de entrega [2,4]. La observación de las fases por microscopia electrónica y su identificación por medio de microanálisis químico (Figura 4), han permitido la identificación de los precipitados $\operatorname{Al}_{6}(\mathrm{Mn}, \mathrm{Fe}) \mathrm{y}(\beta)$ $\mathrm{Al}_{3} \mathrm{Mg}_{2}$ en la estructura, coincidiendo con el resultado obtenido a través de simulación termodinámica de transformaciones de fase en equilibrio y que, corresponde al tipo de (segundas) fases esperadas en aleaciones de aluminio AA5083 [12-14]. Los precipitados intermedios del tipo ( $\beta$ ) $\mathrm{Al}_{3} \mathrm{Mg}_{2}$ se encuentran bordeando los contornos de grano, mientras que los precipitados de mayor tamaño $\mathrm{Al}_{6}(\mathrm{Mn}, \mathrm{Fe})$ aparecen dispersos ocupando posiciones intergranulares, preferentemente [14]. Cómo se muestra en la identificación experimental de los precipitados intermedios, la fase $\mathrm{Al}_{3} \mathrm{Fe}$ no fue encontrada y su ausencia es coincidente con la baja fracción másica obtenida a través de la simulación termodinámica computacional (Figura 5) y es coherente con la literatura, la cual justifica su ausencia debido a la presencia de Mn [15]

El predominio de la fase $\beta\left(\mathrm{Al}_{3} \mathrm{Mg}_{2}\right)$ en los contornos de grano del material en estado de entrega, como se observa experimentalmente en la figura 4, sugiere la activa participación de estos en el proceso de recristalización estática [15]. Sin embargo, se observa del diagrama de fracción de fases para esta aleación, que la fase en mención solubiliza a $238^{\circ} \mathrm{C}$, hecho que coincide con la reducción de la dureza observada en la Figura 8 [16]. Ya que los precipitados de $\mathrm{Al}_{6}(\mathrm{Mn}, \mathrm{Fe})$ solubilizan a alta temperatura $\left(639^{\circ} \mathrm{C}\right)$, de acuerdo con los campos de estabilidad observados en la Figura 5, se espera que este tipo de precipitados intermedios pueda influir sobre el proceso de recristalización durante los ciclos térmicos aplicados, principalmente por su 
gran tamaño y estabilidad [16]. De acuerdo con el comportamiento de la evolución de las dimensiones largo y ancho del grano en función de la temperatura del tratamiento isotérmico, mostrado en la Figura 7, se observa que el cambio en las dimensiones es heterogéneo, siendo más abrupto para la dimensión del largo. Es muy probable que esta heterogeneidad esté asociada a la no disolución de precipitados $\mathrm{Al}_{6}(\mathrm{Mn}, \mathrm{Fe})$ que se encuentran en el contorno de grano con un tamaño suficiente para convertirse en sitios dispersos de nucleación de la recristalización $[11,16]$.

En general, en la Figura 8 se observa para todas las direcciones un incremento de la dureza a partir de $100^{\circ} \mathrm{C}$, con un pico máximo a $150^{\circ} \mathrm{C}$ hasta un nuevo cambio en $180^{\circ} \mathrm{C}$ con posterior reducción gradual hasta $300^{\circ} \mathrm{C}$, reduciéndose abruptamente desde esta última temperatura en adelante. Una explicación plausible para el incremento observado podría asociarse con las coincidencias en el cambio de tamaño de grano advertidos en el intervalo entre $100^{\circ} \mathrm{C}$ a $180^{\circ} \mathrm{C}$, donde ocurre la recristalización en el material, lo que es coincidente con algunas observaciones experimentales [13, 15-16]. No obstante, de acuerdo con el termograma DSC, mostrado en la Figura 9, existe una primera reacción endotérmica que inicia próxima a los $50^{\circ} \mathrm{C}$ y finaliza en torno de los $166^{\circ} \mathrm{C}$, alcanzado su valor pico en $70^{\circ} \mathrm{C}$. El inicio de esta reacción endotérmica, probablemente está asociada a la disolución de pequeños conglomerados de magnesio en el material, coligados a entramados de dislocaciones, tal como se prevé en este tipo de material altamente deformado [14].

El intervalo de temperaturas de la reacción endotérmica mostrada en la Figura 9, contiene a las temperaturas de reducción del tamaño de grano y el valor máximo de durezas. Seguida de la reacción endotérmica sobreviene una región de reacción exotérmica cuyo valor máximo es observado a $\sim 166^{\circ} \mathrm{C}$ y cuya extensión total va hasta casi $\operatorname{los} 250^{\circ} \mathrm{C}$, coincidente con el intervalo de temperaturas donde se ha remarcado la disolución de la fase $\beta$. El análisis anterior, permite deducir que el inicio del proceso de recristalización del material estudiado está dentro del intervalo entre $150^{\circ} \mathrm{C}$ y $180^{\circ} \mathrm{C}$, siendo un valor más bajo que el reportado en la literatura para este tipo de materiales [13-14]. Es probable asociar la reducción de la temperatura de inicio de la recristalización con el particular estado de fuerte deformación del material en su condición -H116 [15].

Según el termograma DSC, después de los $250^{\circ} \mathrm{C}$ prácticamente es imperceptible alguna reacción que determine cambios importantes en la estructura del material. No es claramente perceptible la disolución de la fase $\beta\left(\mathrm{Al}_{3} \mathrm{Mg}_{2}\right)$ en la Figura 9, la cual debía estar próxima a la temperatura de $238^{\circ} \mathrm{C}$. Sin embargo, después de la reacción endotérmica, aparece un pico de reacción exotérmica con el valor máximo a $166^{\circ} \mathrm{C}$. Esta reacción puede contener la transformación de la fase $\beta$, dada su extensión hasta los $250^{\circ} \mathrm{C}$, aproximadamente. Esta última observación refuerza la idea de que la reducción brusca de la dureza observada a partir de la temperatura de $300^{\circ} \mathrm{C}$ (Figura 8) y en temperaturas posteriores se deba, principalmente al crecimiento del grano después de la disolución de la fase $\beta$, a pesar de la presencia de precipitados de $\mathrm{Al}_{6}(\mathrm{Mn}, \mathrm{Fe})[15,19]$. Algunos autores han observado que, con los cambios de temperatura posteriores a $\operatorname{los} 300^{\circ} \mathrm{C}$, los precipitados del tipo $\mathrm{Al}_{6}(\mathrm{Mn}, \mathrm{Fe})$ pueden modificar su morfología de romboidal a la de dispersoides tipo bastón, o descomponerse en la fase metaestable $\mathrm{Al}_{12} \mathrm{Mn}$, insinuando este hecho que, tal vez, desde esta temperatura y posteriores esta fase ya no sea eficaz para frenar la migración de contornos de grano [14,21-23]. Los ciclos térmicos tienen un importante efecto sobre las propiedades mecánicas y el comportamiento a la corrosión de esta aleación $[20,24-25]$.

\section{CONCLUSIONES}

La variación del tamaño de grano y de la dureza en la aleación de aluminio AA5083-H116 bajo el efecto de diversos tratamientos isotérmicos han sido estudiadas. De acuerdo con las observaciones experimentales realizadas en este trabajo, se concluye que los procesos de recristalización estáticos en estas aleaciones se inician dentro de un intervalo de temperaturas entre $150^{\circ} \mathrm{C}$ y $180^{\circ} \mathrm{C}$ presentándose como un proceso heterogéneo en la estructura del material, con su máxima manifestación alrededor de los $150^{\circ} \mathrm{C}$. La heterogeneidad del proceso de recristalización está ligada al alto nivel de deformación del material debido al proceso de conformación plástica, a la disolución de la fase $\beta$ y a la presencia de precipitados intermedios del tipo $\mathrm{Al}_{6}(\mathrm{Mn}$, $\mathrm{Fe})$ que permanecen estables, incluso a altas temperaturas. La caída abrupta de la dureza a partir de $300^{\circ} \mathrm{C}$ está asociada al crecimiento del grano, mucho más que a la participación concomitante de precipitados intermedios en este comportamiento.

\section{AGRADECIMENTOS}

Los autores de este trabajo agradecen a la Universidad Autónoma del Caribe por el apoyo financiero a este trabajo a través de los proyectos de convocatoria interna CONV-I-004-P010 y No 13 de 2011. Agradecemos al Dr. Conrado Afonso Ramos Moreira, de la Universidad Federal de São Carlos, Brasil por sus gentiles aportes. Así mismo, agradecemos al personal del laboratorio nacional de nanotecnología LNNano del Centro 
nacional de Pesquisa en Energía y materiales (CNPEM) de Brasil, por su invaluable ayuda y aportes en esta investigación.

\section{BIBLIOGRAFIA}

[1] DAVIS J.R., Aluminum and Aluminum Alloys book. ASM specialty handbook, 1 ed., ASM International, cap $1,1993$.

[2] TOTTEN, G.E, MACKENZIE, D.S., Handbook of aluminum v. 1 Physical Metallurgy and Processes, 1 ed., New York, Marcel Dekker Inc., Cap. 1., 2003

[3] SCHWEITZER, P.A., Metallic Materials Physical, Mechanical, and Corrosion Properties, Marcel Dekker, Inc, CRC Press., 2003.

[4] RANDOLPH KISSELL, J., FERRY, R.L., Aluminum Structures: A guide to their specifications and design, New York, Jhon Wiley \& Sons, Inc., 2002.

[5] ZANDER J., SANDSTRÖM, R.S., "Modelling technological properties of commercial wrought aluminium alloys", Materials \& Design, v. 30, n. 9, pp. 3752-3759, 2009.

[6] PADILHA, A.F., SICILIANO, F., Encruamento, recristalização, crescimento de grão e textura, 3 ed., São Paulo, ABM, 232p., 2005.

[7] DOHERTY R.D., et al., "Current issues in recrystallization: a review", Materials Science and Engineering $A$, v. 238, n. 2, pp. 219-274, 1997

[8] HUMPHREYS, F.J., "Modelling mechanisms and microstructures of recrystallisation", Materials Science and Technology, v. 8, n. 2, pp. 135-144, 1992.

[9] MA, T., DEN OUDEN, G., "Softening behaviour of Al-Zn-Mg alloys due to welding", Materials Science and Engineering A, v. 266, n. 1-2, pp. 198-204, 1999.

[10] FU, G., TIAN, F., WANG, Y.H., "Studies on softening of heat-affected zone of pulsed-current GMA welded Al-Zn-Mg alloy", Journal of Materials Processing Technology, v. 180, n. 1-3, pp. 216-220, 2006.

[11] ASTM E-112-13. Standard Test Methods for determining average grain size. American Standard Test Methods International. DOI: 10.1520/E0112-13. West Conshohocken, 2013.

[12] BELOV, N., ESKIN, D., AKSENOV, A., Multicomponent Phase Diagrams. Applications for commercial aluminum alloys, 1 ed., Elsevier, 2005.

[13] FULLER, C., MAHONEY, M., "The Effect of Friction Stir Processing on 5083-H321/5356 Al Arc Welds: Microstructural and Mechanical Analysis", Metallurgical and Materials Transactions A, v. 37A, pp. 3605-3615, 2006.

[14] KANNAN, K., JOHNSON, C., HAMILTON, C., "A study of superplasticity in a modified 5083 Al-MgMn alloy", Metallurgical and materials transactions: A, v. 29A, n. 4, pp. 1211-1220, 1998.

[15] VETRANO, J.S., BRUEMMER, S.M., PAWLOWSKI, L.M., et al., "Influence of the particle size on recrystallization and grain growth in Al-Mg-X alloys", Materials Science and Engineering A, v. 238, pp. 101107, 1997.

[16] OGUOCHA, I.N.A., O.J. ADIGUN, S. YANNACOPOULOS, "Effect of sensitization heat treatment on properties of Al-Mg alloy AA5083-H116", Journal of Materials Science, v. 43, n. 12, pp. 4208-4214, 2008.

[17] RIOS, P.R., "Overview n. 62: A theory for grain boundary pinning by particles", Acta Metallurgica, v. 35, n. 12, pp. 2805-2814, 1987.

[18] DRIVER, J.H., PAPAZIAN, J.M., "Microstructural effects of the cyclic and monotonic hardening of Al5Mg", Materials Science and Engineering, v. 76, pp. 51-56, 1985.

[19] VERDIER, M., ET AL., "Dislocation densities and stored energy after cold rolling of Al-Mg alloys: investigations by resistivity and differential scanning calorimetry", Scripta Materialia, v. 37, n. 4, pp. 449454, 1997.

[20] OGUOCHA, I. N. A., ADIGUN, O. J., YANNACOPOULOS, S., "Effect of sensitization heat treatment on properties of Al-Mg alloy AA5083-H116", Journal of Materials Science, v. 43, pp. 4208-4214, 2008.

[21] ZHOU, F., ET AL., "Microstructural evolution during recovery and recrystallization of a nanocrystalline Al-Mg alloy prepared by cryogenic ball milling", Acta Materialia, v. 51, pp. 2777-2791, 2003.

[22] RATCHEV, P., VERLINDEN, B., VAN HOUTTE, P., "Effect of preheat temperature on the orientation relationship of $(\mathrm{Mn}, \mathrm{Fe}) \mathrm{Al}_{6}$ precipitates in an AA5182 Aluminum-Magnesium alloy", Acta metallurgica et materialia, v. 43, n. 2, pp. 621-629, 1995.

[23] NUÑEZ, E.E., Influencia del aporte térmico en la soldabilidad de la aleación AA5083-H116 con proce- 
so GMAW-P automatizado, Tesis doctoral, Universidad del Norte, Barranquilla, Colombia, 2013.

[24] MENG, CH., ZHANG, D., CUI, H., et al., "Effect of stabilizing treatment on the intergranular corrosion behavior of high strength Al-Mg alloys", Materials Science Forum., v. 794-796, pp.253-258, 2014.

[25] CHEN, R-Y., CHU, H-Y., LAI, CH-CH., et al. "Effects of annealing temperature on the mechanical properties and sensitization of 5083-H116 aluminum alloy", Proceedings of the Institution of Mechanical Engineers, Part L: Journal of Materials Design and Applications, v. 229, n 4, p. 339-346, 2015. 